\title{
Observations of flux rope formation prior to coronal mass ejections
}

\author{
Lucie M. Green ${ }^{1}$ and Bernhard Kliem ${ }^{2}$ \\ ${ }^{1}$ Mullard Space Science Laboratory, UCL, Holmbury St. Mary, Dorking, Surrey, UK ${ }^{2}$ \\ University of Potsdam, Institute of Physics \& Astronomy, Potsdam, Germany
}

\begin{abstract}
Understanding the magnetic configuration of the source regions of coronal mass ejections (CMEs) is vital in order to determine the trigger and driver of these events. Observations of four CME productive active regions are presented here, which indicate that the pre-eruption magnetic configuration is that of a magnetic flux rope. The flux ropes are formed in the solar atmosphere by the process known as flux cancellation and are stable for several hours before the eruption. The observations also indicate that the magnetic structure that erupts is not the entire flux rope as initially formed, raising the question of whether the flux rope is able to undergo a partial eruption or whether it undergoes a transition in specific flux rope configuration shortly before the CME.
\end{abstract}

Keywords. Sun: coronal mass ejections (CMEs), Sun: activity

\section{Introduction}

Twisted bundles of magnetic field lines known as flux ropes are central to all models of coronal mass ejections. They are invoked as being present either before the eruption onset (e.g. Török \& Kliem 2005) or being formed during the eruption itself (e.g. Antiochos et al. 1999). In light of this, one way to discriminate between these two sets of CME models is to determine the pre-eruption magnetic configuration. However, there is an inherent difficulty in the confident identification of flux ropes as it is not currently possible to directly measure the magnetic field above the photosphere/chromosphere. Instead, proxies for the presence of flux ropes need to be used, either from solar observations or from reconstructions of the coronal magnetic field using the photospheric field as the boundary condition.

One well developed observational approach to investigate the pre-eruption magnetic configuration is to study so-called sigmoidal active regions (Rust \& Kumar 1996). Sigmoidal regions contain S shaped EUV and X-ray emission structures and are regions that have a very high likelihood of producing a coronal mass ejection. Sigmoids are seen as predictors of an eruption (Canfield et al. 1999). The sigmoid can be $\mathrm{S}$ or reverse $\mathrm{S}$ shaped, depending on the chirality of the magnetic field in which it forms (Pevtsov et al. 1997). Rust \& Kumar (1996) suggested a link between sigmoids and kinking flux ropes, which has been a lively area of research ever since. Theoretical expectations from modelled flux ropes show that layers of enhanced current, and presumably heating, are located at the interface between a flux rope and its surrounding magnetic arcade (Titov \& Démoulin 1999). These layers should appear $\mathrm{S}$ shaped when viewed from above, building the case that sigmoids represent flux ropes in the solar atmosphere.

Recent investigations into the magnetic configuration of sigmoids have shown support that, at least a sub-set of sigmoids, do indeed indicate the presence of a magnetic flux rope. Green \& Kliem (2009) showed that continuous S shaped threads that make an inverse crossing of the photospheric polarity inversion line (PIL) in their centre strongly 
suggest field lines that spiral around the flux rope. These threads additionally cross the PIL in the normal direction in the sigmoid's elbows. They trace field lines at the periphery of the rope and are likely to extend down to the lower atmosphere, where they form bald patches.

As well as forming in active regions, sigmoids have been observed on a smaller scale in X-ray bright points (Mandrini et al. 2005) and on a larger scale in the quiet Sun (Jiang et al. 2007). It has been suggested that there are different types of sigmoid relating to their lifetime (transient and long-lived) or detailed observational appearance (continuous $\mathrm{S}$ threads or double J's that overall look S shaped) (Pevtsov 2002a).

In summary, flux ropes can be investigated in the solar atmosphere prior to a coronal mass ejection by using observations of sigmoids. These features provide an opportunity to follow the evolution of the magnetic configuration and investigate how the flux rope forms, the magnetic flux content of the rope and the specific magnetic configuration. In this paper we focus on flux rope formation in a small sample of sigmoidal active regions in the days leading up to a coronal mass ejection.

\section{Observations of flux rope formation}

Here we present the evolution of four active regions; (1) NOAA region 10930 that was observed on the Sun during December 2006, (2) NOAA region 8005 that was seen during December 1996, (3) an un-numbered region that was seen in February 2007 and (4) NOAA active region 10977 that was on the disk during December 2007. This sample includes one region which forms a sigmoid during a flux emergence event and three regions which show sigmoidal structure forming during the decay phase of the region.

\subsection{NOAA active region 10930}

Active region 10930 rotated over the eastern limb of the Sun on 5 December 2006. The region contained a large negative polarity sunspot with a corresponding dispersed and spotless positive magnetic field to the east. Immediately to the south of the negative polarity sunspot was a smaller magnetic bipole. On 10 December 2006, new flux began to emerge in the location of the smaller bipole. The positive polarity of the emerging flux underwent an eastward motion and a strong counter clockwise rotation of up to 540 degrees between 10 and 14 December (Min \& Chae 2009). The positive polarity of the emerging flux was directly next to the pre-existing negative spot meaning that flux cancellation was likely to be taking place.

The XRT/Hinode (Golub et al. 2007) data show that as soon as the flux emergence begins, the magnetic field of the positive sunspot formed connections with the pre-existing negative sunspot producing a magnetic arcade between them. The shear in this arcade field increased rapidly, most likely due to the rapid eastward motion of the emerging flux and its strong counter-clockwise rotation. By 23:15 UT on 11 December the arcade field had taken on the appearance of a 'double J' configuration and by 19:00 UT on 12 December a continuous S shaped structure was seen. See the top row of Fig. 1 for the coronal evolution of this region. On 13 December 2006 a GOES X3.4 class flare occurred in the sigmoidal region at around 02:15 UT. A coronal mass ejection was associated with this flare.

\subsection{NOAA active region 8005}

NOAA active region 8005 had a bipolar magnetic configuration. The region emerged on the far side of the Sun and as it rotated over the limb it was already in its decay phase with dispersed magnetic polarities and no sunspots. The photospheric field exhibited dispersal 
of the magnetic polarities and no episodes of flux emergence. On many occasions opposite polarity fragments approached the active region's polarity inversion line and cancelled. See Green \& Kliem (2009) and Mackay et al. (2011) for details of the evolution of the photospheric magnetic field in this region. In the days leading up to the eruption the active region loops as seen in the soft X-ray data had an overall S shape which evolved from a sheared arcade, to a double J shape (by 19 December 07:24 UT) to a sigmoid with continuous S shaped threads being observed by 10:37 UT on 19 December. See the second from top row of Fig. 1 for the coronal evolution of this region. The active region produced an eruption and a GOES C2.3 class flare on 19 December 1996 at 15:21 UT.

\subsection{February 2007 region}

In February 2007 a sigmoid was observed in a very dispersed bipolar active region that had no NOAA number assigned to it. The region produced a coronal mass ejection on 12 February 2007 beginning around 07:00 UT. There was no flare emission associated with this eruption. In the days leading up to the eruption the active region evolved from a double J configuration to having continuous $\mathrm{S}$ shaped loops by end of the day on 11 February 2007. See the second row from the bottom of Fig. 1 for the coronal evolution of this region. The evolution of the photospheric magnetic field showed ongoing dispersal and episodes of flux cancellation at the polarity inversion line. For more details on the evolution of this active region see Savcheva et al. (2012).

\subsection{NOAA active region 10977}

NOAA active region 10977 had a bipolar configuration and the whole lifetime of the active region was observed from emergence to dispersal into the surrounding quiet sun. The emergence began on 3 December 2007 and the flux concentrations began to disperse on 4 December 2007. During the decay phase of the active region the photospheric field was dominated by ongoing dispersal, an elongation of the polarities in the north-south direction and cancellation of flux along the polarity inversion line.

Hinode/XRT data show that the active region loops appeared to have relatively little shear during the emergence phase and into 5 December after the emergence had ceased. The shear began to build up during the decay phase when the loops became much more aligned to the polarity inversion line. Early on 6 December the region appeared as a region of double $\mathrm{J}$ shaped loops with some remnant arcade field in the south. Then, by 6 December 15:50 UT continuous S shaped sigmoidal threads were seen in the region. See the bottom row of Fig. 1 for the coronal evolution of this region. On 7 December at around 04:20 UT the region produced a coronal mass ejection. For a more detailed description of the evolution of this active region see Green et al. (2011).

\section{Filament formation}

All of the above active regions exhibited the formation of a filament along the polarity inversion line where the sigmoid formed. Big Bear Solar Observatory H-alpha data show that a filament was present in NOAA active region 10930 by 11 December 18:00 UT. In NOAA region 8005 the filament had formed by 18 December 17:40 UT. In the unnamed region seen on the disk in February 2007, Kanzelhöhe Observatory data show that there was a filament present by 10 February 2007 12:00 UT. In NOAA region 10977 Hinode/SOT H-alpha data show that the filament was forming by 5 December around 20:00 UT. The filaments in all these regions formed during the phase where the coronal arcade was becoming more sheared. The presence of a filament indicates low lying loops that have dips, or twisted field lines which can support the dense plasma against gravity. 

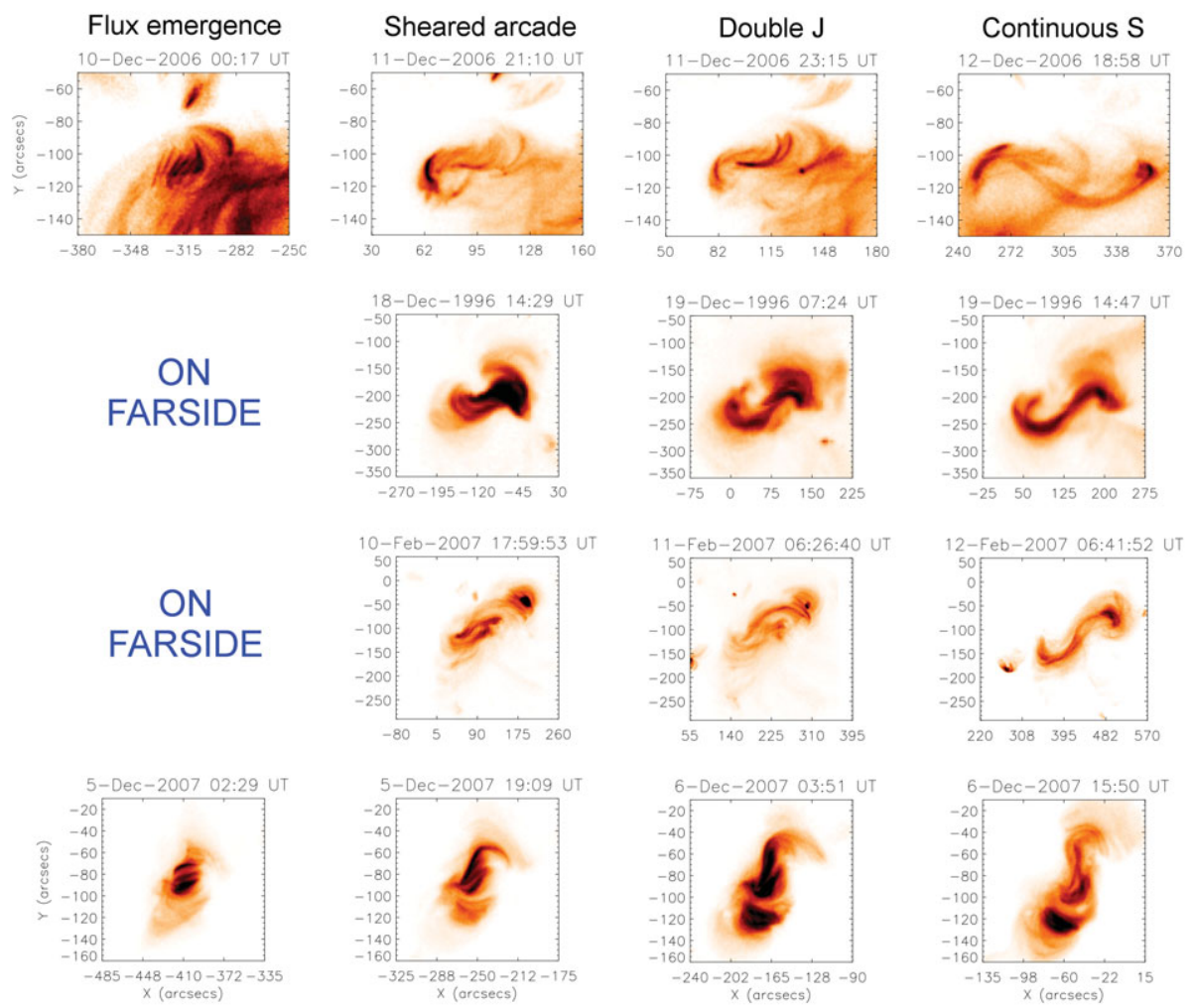

Figure 1. Evolution of sigmoidal active regions showing the three phases of evolution from flux emergence, to sheared arcade, to 'double J' shaped loops and then finally the continuous S shaped threads of the sigmoid. Top row: NOAA region 10930. Second row from the top: NOAA region 8005. Second row from the bottom: un-numbered region observed on the disk during February 2007. Bottom row: NOAA region 10977.

\section{Coronal mass ejections from the sigmoidal regions}

The observation of the sigmoid allows the magnetic configuration to be probed and a time for the formation of the flux rope to be identified. The $\mathrm{S}$ shaped field lines can indicate the presence of magnetic field lines with dips which suggests the presence of a flux rope, even if it is not fully formed. The time between sigmoid (and flux rope) formation and the onset of the coronal mass ejection is given in Fig. 2. To increase the study size this figure also includes the sigmoid and coronal mass ejection that occurred in the bright point study of Mandrini et al. (2005) and the sigmoidal region NOAA 11047 that produced a coronal mass ejection and that was studied in Savcheva et al. (2012). The colours indicate whether the region was observed from the emergence phase, and hence whether all activity is seen, or whether the region emerged on the far-side so that aspects of the photospheric and coronal evolution may have been missed. In all cases the flux rope exhibits a stable phase of several hours between its formation and eruption.

In all four regions of this study the coronal mass ejection does not involve the filament material, as has been previously shown when sigmoidal active regions produce coronal mass ejections (Pevtsov 2002b). The events presented here show that the S shaped loops also do not rise during the eruption. Instead, the erupting structure in three out of the 


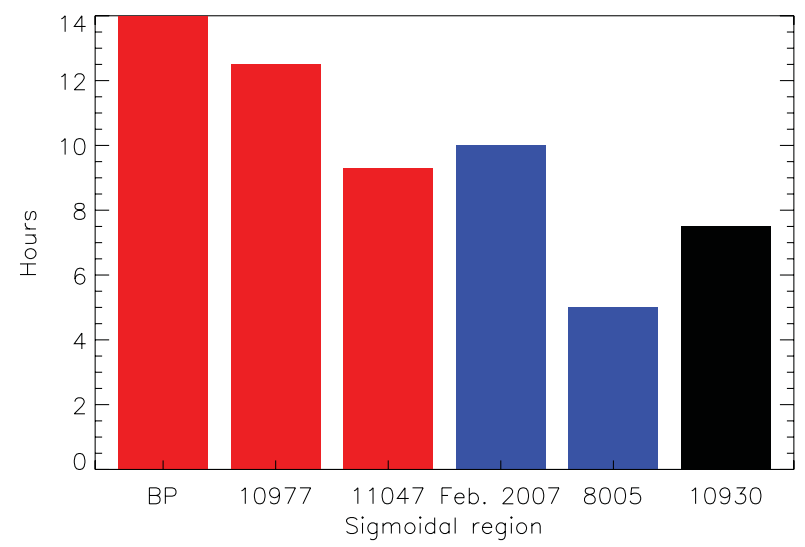

Figure 2. Bar chart showing the time between first observation of continuous S shaped loops in the regions and the time of the coronal mass ejection. The colours indicate different region characteristics. Red: isolated bipolar regions that have been followed from emergence to eruption and which produce the sigmoid and the eruption in their decay phase. Blue: isolated bipolar regions that emerged on the far side of the Sun and which produced the sigmoid and eruption during their decay phase. Black: a multipolar active region tracked from a flux emergence episode to the sigmoid formation and eruption and which formed the sigmoid during the flux emergence phase.

four sigmoidal regions is a faint linear or loop-like feature that rises up as the flare loops brighten underneath. See Fig. 3 in Su et al. (2007) for region 10930, McKenzie \& Canfield (2008) for the case in February 2007 and Green et al. (2011) for region 10977.

\section{Discussion}

There is growing observational support that a flux rope is present before the onset of a coronal mass ejection in some cases (see Aulanier et al. 2010, Green et al. 2011 and references therein). Active regions that show a sigmoidal structure have been fruitful in revealing these flux ropes and their formation mechanism. The observations presented here strongly suggest that flux ropes in the solar atmosphere are built by the flux cancellation mechanism proposed by van Ballegooijen \& Martens (1989). Flux cancellation involves reconnection low down in the solar atmosphere between converging opposite polarity fragments in a sheared arcade. The evolution of the coronal configuration is driven by the motions of the photospheric plasma and in this study is seen to pass through three stages as arcade field evolves into a flux rope. During the first stage shear in the coronal arcade field increases due to photospheric motions associated with flux emergence or flux dispersal and flux cancellation. Filaments start to form during this stage. During stage two there is an accumulation of a significant amount of axial flux running along the polarity inversion line as flux cancellation, further shearing and/or rotation of the magnetic polarities takes place. Remnant arcade field takes on the appearance of two J's either side of this axial flux. In stage three, flux cancellation produces field lines that are twisted around the axial flux and which contribute poloidal flux to the rope. This flux cancellation scenario appears to be relevant in dispersed and isolated bipolar active regions in their decay phase, where the flux rope forms along the polarity inversion line of the bipole, or during the flux emergence phase of a multipolar active region where the flux rope forms along the polarity inversion line between neighbouring bipoles which are butted up against each other. The observations of the evolutionary phases suggest that 
sigmoids do not exhibit different types, rather their appearance evolves as the magnetic configuration changes.

The details of the eruptions from these regions are also important for understanding the pre-eruption magnetic configuration and the aspects of this configuration that are involved in the coronal mass ejection. The eruptions from these regions do not involve either the continuous S shaped threads or the filament. Since both are likely to involve field lines that are located in, or extend down to, the dense plasma of the lower atmosphere, it is not surprising that they are immobile. The flux rope cannot erupt in its entirety in this situation. The observations show that the structure which does erupt is instead a collection of loops that connect between the elbows of the $\mathrm{S}$ shaped threads and which can be seen before the onset of the eruption. These loops have been called a linear feature and tentatively been associated with the core of the erupting flux rope in Green et al. (2011). However, in Aulanier et al. (2010) the same feature is interpreted as being a consequence of heating in a current shell above the rope and called an erupting loop-like feature. Such an erupting structure was also seen by Moore et al. (2001) but has become more frequently observed with Hinode/XRT due to its large dynamic range and also with SDO due to the increased plasma temperature coverage. See, for example, Liu et al. (2010) and Zharkov et al. (2011).

The observations presented here suggest that either the flux rope is able to partially erupt, allowing the accumulated axial flux to escape and become the erupting linear feature, whilst leaving the flux associated to the S shaped threads and filament behind. In other cases the flux rope may evolve prior to the eruption so that its underside is detached from the lower atmosphere, allowing it to erupt fully, as demonstrated in the simulations of Aulanier et al. (2010).

\section{References}

Antiochos, S. K., DeVore, C. R., \& Klimchuk, J. A. 1999, ApJ, 510, 484

Aulanier, G., Török, T., Démoulin, P., \& DeLuca, E. 2010, ApJ, 708, 314

Canfield, R. C., Hudson, H. S., \& McKenzie, D. E. 1999, GeoRL, 26, 627

Golub, L., Deluca, E., Austin, G., et al. 2007, Solar Phys., 243, 63

Green, L. M. \& Kliem, B. 2009, ApJ, 700, L83

Green, L. M., Kliem, B., \& Wallace, A. J. 2011, A\& $A, 526,2$

Jiang, Y., Chen, H., Shen, Y., Yang, L., \& Li, K. 2007, Solar Phys., 240, 77

Liu, R., Liu, C., Wang, S., Deng, N., \& Wang, H. 2010, ApJ, 725, 84

Mackay, D. H., Green, L. M., \& van Ballegooijen, A. A. 2011, ApJ, 729, 97

Mandrini, C. H., Pohjolainen, S., Dasso, S., et al. 2005, A\&A A, 434, 725

McKenzie, D. E. \& Canfield, R. C. 2008, A\&A, 481, 65

Min, S. \& Chae, J. 2009, Solar Phys., 258, 203

Moore, R. L., Sterling, A. C., Hudson, H. S., \& Lemen, J. R. 2001, ApJ, 552, 833

Pevtsov, A. A. 2002a, in P.C.H. Martens and D. Cauffman (eds.), "Multi-Wavelength Observations of Coronal Structure and Dynamics - Yohkoh 10th Anniversary Meeting", p.125

Pevtsov, A. A. 2002b, Solar Phys., 207, 111

Pevtsov, A. A., Canfield, R. C., \& McClymont, A. N. 1997, ApJ, 481, 973

Rust, D. M. \& Kumar, A. 1996, ApJ, 464, 199

Savcheva, A. S., Green, L. M., van Ballegooijen, A. A., \& DeLuca, E. 2012, ApJ, 759, 105

$\mathrm{Su}$, Y., Golub, L., \& van Ballegooijen, A. A., et al. 2007, PASJ, 59, 785

Titov, V. S. \& Démoulin, P. 1999, A\&A A, 351, 707

Török, T. \& Kliem, B. 2005, ApJ, 630, L97

van Ballegooijen, A. A. \& Martens, P. C. H.. 1989, ApJ, 343, 971

Zharkov, S., Green, L. M., Matthews, S. A., \& Zharkova, V. V. 2011, ApJ, 741, L35 